CORRECTION

\title{
Correction: Genetic variation in $C R H R l$ is associated with short-term respiratory response to corticosteroids in preterm infants at risk for bronchopulmonary dysplasia
}

Tamorah Lewis ${ }^{1}$, William Truog ${ }^{1}$, Mike Norberg ${ }^{1}$, Philip L. Ballard ${ }^{2}$ and Dara Torgerson ${ }^{2}$ for the TOLSURF Study Group

Pediatric Research (2019) 85:731; https://doi.org/10.1038/s41390-019-0314-y

Correction to: Pediatric Research; https://doi.org/10.1038/s41390018-0235-1; published online 22 November 2018

In the original version of this article, the Supplementary Information file contained incorrect reference numbers.
'Supplemental Table S1' has now been replaced with the corrected version, in which the correct reference numbers are cited. The authors would like to apologise for this error.

${ }^{1}$ Children's Mercy Hospital, Department of Pediatrics, University of Missouri Kansas City School of Medicine, Kansas City, MO, USA and ${ }^{2}$ Department of Pediatrics, University of California San Francisco, San Francisco, CA, USA

Correspondence: Tamorah Lewis (trlewis@cmh.edu)

Published online: 7 February 2019 